\title{
Política exterior, proyectos e integración en los gobiernos kirchneristas
} (2003-2015)

\author{
Gastón Ángel Varesi*
}

\begin{abstract}
Resumen
El artículo se propone analizar la política exterior de los gobiernos kirchneristas en vinculación a las dinámicas más generales de la construcción de hegemonía a nivel Estado y del régimen de acumulación de capital. En este camino, observamos la conformación del proyecto político-económico, con un componente clave en el discurso presidencial en tanto base ideológica que constituye una lectura común del pasado y del futuro a construir, observando la interrelación de la escala nacional con la internacional en el debate por un nuevo orden global. Allí, indagamos el rol que cumple el proceso de integración regional, a partir de la caracterización de la guerra de posiciones que atraviesa el continente en el naciente siglo XXI. Con esta propuesta, emprendemos un análisis de las relaciones de fuerzas internacionales que nos permita enmarcar y comprender diversos hitos de la política exterior e interior del kirchnerismo entre 2003 y 2015, donde las categorías de autonomía, pueblo, nación y neo-desarrollismo se ligan a las de regionalización, multilateralismo y multipolaridad, en una lectura que articula múltiples dimensiones y escalas.
\end{abstract}

Palabras claves: política exterior - kirchnerismo - hegemonía - neo-desarrollismo - integración regional

\section{Foreign Policy, projects and regional integration in the Kirchnerists administrations (2003-2015)}

\begin{abstract}
The article aims to analyze the foreign policy of Kirchnerist administrations in connection with the general dynamics of the construction of hegemony at the State level and the regime of accumulation. In this way, we observe the conformation of the political-economic project, with a key component in the presidential discourse as an ideological base that constitutes a common reading of the past and the future to be built, observing the interrelation of the national scale with the international one in the debate for a new global order. There, we investigate the role of the process of regional integration, based on the characterization of the war of positions that is being developed through the continent in the nascent 21 st century. With this proposal, we undertake an analysis of international relations of force that allows us to understand various milestones of the foreign and domestic policy of Kirchnerism between 2003 and 2015 , where the categories of autonomy, people, nation and neo-developmentalism are linked to regionalization, multilateralism and multipolarity, in an interpretation that articulates multiple dimensions and scales.
\end{abstract}

Keywords: foreign policy, Kirchnerism, hegemony, neo-developmentalism, regional integration.

TRABAJO RECIBIDO: 18/02/2019 TRABAJO ACEPTADO: 08/04/2019

\footnotetext{
* Sociólogo, Doctor en Ciencias Sociales, Universidad Nacional de La Plata (UNLP, Argentina). Investigador asistente del CONICET (IdIHCS-UNLP). Profesor de "Economía, Política y Sociedad en la Argentina Contemporánea" del Doctorado y la Maestría en Ciencias Sociales y la Maestría en Políticas de Desarrollo (UNLP), Profesor Adjunto de "Geografía Económica Argentina" (FAHCE-UNLP). Coordinador de la sede platense del Centro de Estudios y Formación Marxista H. Agosti, (CEFMA).
} 


\section{Introducción}

El presente artículo se inscribe en el marco de la investigación titulada "Modelo de acumulación y hegemonía durante los gobiernos kirchneristas (2003-2015). Políticas económicas, fracciones de clase y antagonismo en Argentina, en el marco del nuevo contexto latinoamericano" y se aboca al desarrollo de uno de sus objetivos específicos. El mismo busca aportar al análisis de la política exterior del Gobierno argentino, enfocando en el proceso de integración latinoamericano, con el fin de articular la escala nacional con la escala internacional, viendo sus particularidades e interrelaciones. Para ello se ha desplegado una estrategia teóricometodológica que combina diversas técnicas cualitativas y cuantitativas como el análisis de documentos, discursos, leyes e indicadores socio-económicos.

En este camino, el artículo comienza con un primer apartado donde se aborda la hegemonía kirchnerista y el marco de las relaciones de fuerzas internacionales, delineando los principales conceptos teóricos y el estado de la cuestión. Trazando un recorrido de lo general a lo particular, contextualizamos la situación internacional en el marco del "nuevo" imperialismo y su articulación neoliberal como orden en disputa, para situarnos en el creciente contra-peso multipolar global, con nuevas potencias emergentes y la nueva situación en América Latina. Allí, abordamos la guerra de posiciones que comenzó a caracterizar a la región, a partir del "giro a la izquierda" y la conformación del bloque popular latinoamericano, signado por el surgimiento y unidad compleja de proyectos socialistas y neo-desarrollistas. A continuación, descendemos a la escala nacional, donde analizamos la hegemonía kirchnerista, indagando su proyecto así como los aspectos nodales de la política exterior, teniendo en el discurso presidencial una fuente valiosa para su aproximación político-ideológica.

En un apartado posterior, nos centramos en algunos hitos de la política exterior argentina durante el gobierno de Néstor Kirchner (NK). Observamos el rechazo al ALCA y la apuesta latinoamericana, con el MERCOSUR como base. La política de desendeudamiento, con la salida del default y pago al Fondo Monetario Internacional (FMI). Y la contraposición en el discurso presidencial entre el capitalismo "serio", de cuño neo-desarrollista, versus el Consenso de Washington que consolidó la matriz neoliberal. Asimismo, indagamos la apuesta por el multilateralismo y su articulación con el reclamo por Malvinas, el rol de las Misiones de Paz de la ONU, la lucha contra el terrorismo y la defensa de los Derechos Humanos (DDHH).

En el siguiente apartado, abordamos los gobiernos de Cristina Fernández de Kirchner (CFK) destacando diversos factores. En primer lugar, indagamos el rol de las categorías de pueblo y nación en la estrategia hegemónica y su vinculación a la disputa de proyectos y regímenes de acumulación. Avanzamos a la caracterización de los mismos a la luz de la crisis mundial y las propuestas divergentes de salida a la crisis. Luego, observamos la búsqueda para profundizar la integración regional con nuevos instrumentos como Unión Suramericana de Naciones (UNASUR) y Comunidad de Estados Latinoamericanos y Caribeños (CELAC). En este punto, se analiza un cambio en el contexto de relaciones de fuerzas internacionales a partir de la contra-ofensiva imperialista sobre la región. Al asedio económico, como el caso de los fondos "buitres", con el respaldo de Estados Unidos (EEUU), se le suma el avance de los "golpes suaves". Asimismo, atendemos a las estrategias de respuestas frente a este escenario, tales como el impulso de la Resolución de reestructuración de deudas en la Organización de las Naciones Unidas (ONU), la unidad regional bajo el liderazgo de los gobiernos populares y los acuerdos estratégicos con China y Rusia para reforzar la multipolaridad.

Finalmente, se esbozan las conclusiones delineando los principales factores que vinculan las múltiples dimensiones y escalas de análisis, evaluando los alcances de la estrategia hegemónica, el rol de la autonomía y la integración así como los desafíos del multilateralismo y la multipolaridad global, abriendo interrogantes para la continuidad de la investigación. 


\section{La hegemonía kirchnerista y su contexto internacional}

El kirchnerismo accedió al Gobierno y se configuró como fuerza política tras la crisis de 2001. Esta crisis constituyó el caso nacional de colapso político, económico y social del régimen neoliberal, que había comenzado a instituirse a partir de las reformas estructurales desplegadas por la dictadura en 1976 y que tuvo su momento más acabado en los años 90.

Este período materializó en la escala nacional procesos de largo aliento que venían modificando las relaciones de fuerzas internacionales. Para su análisis, siguiendo a Gramsci (2003), deben abordarse dos dimensiones: a) las características orgánicas del modo de producción globalmente dominante y b) las potencias mundiales y la conformación de bloques de Estados hegemónicos, indagando su relación con las potencias y bloques menores, abriendo paso al estudio de las dinámicas del imperialismo, los procesos de integración y las luchas por la soberanía.

En este camino, recuperamos la lectura que propone Harvey (2004) para el período en cuestión, donde se consolida un "nuevo" imperialismo basado en la hegemonía norteamericana $\mathrm{y}$ apoyado por los organismos financieros internacionales, que promovieron al neoliberalismo como nueva ortodoxia económica. Esto "implicó un cambio en la correlación de poder dentro de la propia burguesía, en el que los sectores productivos perdieron poder frente a las instituciones del capital financiero. Esto podía usarse para combatir el poder de los movimientos de trabajadores en la reproducción ampliada, ya sea directamente, ejerciendo la supervisión disciplinaria en la producción, o indirectamente, facilitando la mayor movilidad geográfica de todas las formas de capital" (2004:118). Así, el capital financiero cumplió un papel central para esta fase del capitalismo, en la cual se fue gestando un proceso de acumulación por desposesión al ritmo de la proliferación de "temas como los ajustes estructurales impuestos por el FMI, las actividades depredadoras del capital financiero y la pérdida de derechos a través de la privatización" (Harvey, 2004:118). Además, las crisis de endeudamiento externo en los países periféricos fue una punta de lanza para condicionar a los gobiernos a la implementación de aquellas políticas que luego se consolidarían en el denominado Consenso de Washington. Este escenario de relaciones de fuerzas internacionales alcanzó su mayor contundencia con el triunfo de EEUU y sus aliados en la Guerra Fría sobre el bloque soviético, definiendo la globalización como transnacionalización del capital y estableciendo la unipolaridad global, con aspiraciones hegemónicas de sentenciar el "fin de la historia" y el aparente agotamiento de alternativas viables al liberalismo occidental (Fukuyama, 1989).

Sin embargo, no muchos años después, las promesas incumplidas por el nuevo orden global cuyo correlato se expresó en un fuerte incremento de la pobreza, la desocupación, la precarización laboral y la desigualdad, constituyeron un escenario de crisis y de avance de las luchas populares, con particular importancia en América Latina. Así, la dinámica regional comenzó a expresar una genuina guerra de posiciones, concepto que Regalado (2014) recupera de Gramsci para el abordaje de las disputas hegemónicas. Según el autor, esa guerra se libra "entre el imperialismo norteamericano y sus aliados criollos, de una parte, y los movimientos populares y las fuerzas políticas de izquierda y progresistas, de la otra, y un elemento clave de ella es la disputa política y electoral por el control de los gobiernos de la región" (Regalado, 2014:51).

Es allí donde se ubica la llegada de Néstor Kirchner al Gobierno, con la peculiaridad de que en dichas elecciones había salido segundo con el $22 \%$, contando con menos votos que los desocupados que había en país (como luego recordarían en sus discursos tanto él como Cristina Fernández de Kirchner -CFK), y había ganado tras el abandono de su rival, Carlos Menem, al balotaje. Partiendo de tal debilidad de origen y en el contexto de una crisis integral, el kirchnerismo se conformó como fuerza político-hegemónica siguiendo lo que caracterizamos como una doble lógica del populismo: a) mediante la articulación de demandas, conformando su propia identidad en la confrontación con el neoliberalismo y sus figuras diversas como adversario y b) como pacto populista, en el intento de articulación poli-clasista, de intereses de 
fracciones de clases dominantes y subalternas conducidos desde el Estado en la implementación de un régimen de carácter neo-desarrollista (Varesi, 2014).

En este camino, la hegemonía, entendida como conducción política, ideológica y cultural de un grupo social sobre otros, fue construida por el kirchnerismo "planteando todas las cuestiones en torno a las cuales hierve la lucha" (Gramsci, 2003:58), ya que enfrentó los factores persistentes de la crisis de 2001 y se planteó a sí mismo como momento de sutura y superación. Esta articulación de demandas fue sintetizada en sus líderes, primero por NK y luego y simultáneamente por CFK, estableciendo un discurso donde se plasma una lectura común del pasado y de la sociedad a fundar, enunciando la unidad de fines políticos y económicos, permitiéndonos indagar la matriz ideológica que compone todo proyecto políticoeconómico de gobierno, del cual se inspiran las políticas públicas que procuran su materialización.

El análisis de los discursos presidenciales (2003-2015) nos permite precisar un conjunto de factores que hacen a la identidad del kirchnerismo como fuerza hegemónica: la reformulación del peronismo clásico articulado con componentes culturales de la generación de los años 70, la perspectiva de DDHH del progresismo democrático de los 80 y el perfil latinoamericanista ligado al nuevo contexto regional del "giro a la izquierda" en los comienzos del siglo XXI. Asimismo, el proyecto oficial fue cobrando diversas denominaciones como capitalismo "serio", "nacional" o "productivo" o directamente como modelo de crecimiento con inclusión social. Aun definiendo su proyecto como una versión particular dentro de la sociedad capitalista, la definición identitaria erigida en ruptura con el neoliberalismo y el hecho de constituir un gobierno emergente de las luchas contra dicho régimen y de la crisis dejada por éste, acercó al kirchnerismo a otros gobiernos que fueron radicalizando la crítica al orden vigente. Algunos de ellos llegaron a impugnar no sólo el régimen neoliberal sino al capitalismo como modo de producción dominante, proponiendo diversas variantes del socialismo como alternativa: ya no sólo el histórico caso de Cuba, sino también experiencias novedosas como la Venezuela de Chávez y la Bolivia de Evo Morales. De este modo, proyectos neo-desarrollistas y socialistas confluyeron en la conformación de un bloque popular latinoamericano que se constituyó en el sujeto activo del proceso de integración del período.

En este marco, entendemos que la política exterior del kirchnerismo, lejos de responder a necesidades electorales o a una mera primacía de la política interna (De la Balze, 2010; Corigliano, 2011), presentó un conjunto de factores comunes consistentes a lo largo de los años ligados a la concepción del mundo constitutiva del proyecto hegemónico, el cual sin estar exento de condicionantes internos y externos (Busso, 2016; Actis et al, 2017; Busso et al, 2017) presentó regularidades (Simonoff, 2009; 2013) y alcances destacados en un vínculo complejo entre la escala nacional, regional y global. Dentro de los principales factores comunes que atraviesan los tres gobiernos kirchneristas, tanto en materia de iniciativas políticas como del discurso, se destacan los siguientes: 1) Crítica al orden económico imperante (neoliberalismo o "capitalismo anarco-financiero") y propuestas alternativas 2) Integración regional y autonomía; 3) Multilateralismo y multipolaridad; 4) Lucha contra el terrorismo internacional y defensa de los DDHH; 5) Reclamo por Malvinas e Islas del Atlántico Sur. Por cuestiones de espacio y relevancia en relación al objetivo específico abordado, nos centraremos en los tres primeros.

\section{Hitos de política exterior en el gobierno de Néstor Kirchner}

Ya en su discurso de asunción, NK señalaba que "En nuestro proyecto ubicamos en un lugar central la idea de reconstruir un capitalismo nacional que genere las alternativas que permitan reinstalar la movilidad social ascendente" (25/5/2003), para lo cual era preciso "promover políticas activas que permitan el desarrollo y el crecimiento económico del país, la generación de nuevos puestos de trabajo y la mejor y más justa distribución del ingreso. Como se comprenderá el Estado cobra en eso un papel principal, en que la presencia o la ausencia del Estado constituye toda una actitud política" (25/5/2003). 
De este modo, comenzó a cobrar forma un nuevo régimen de acumulación de carácter neo-desarrollista que implicó distintas rupturas en relación al orden neoliberal precedente. En primer lugar, cobró un nuevo énfasis la industria, con un momento destacado de expansión entre 2003 y 2007 al 10,3\% interanual, creciendo incluso por encima del PBI en su conjunto, el cual evolucionó al 8,8\% promedio anual en el mismo período ${ }^{1}$. Esto se vinculó, a su vez, al cambio establecido en los precios relativos, gestado a partir de la regulación de las tarifas de servicios públicos y el control de la tasa de interés, reduciendo los costos, y a la ampliación de la capacidad de consumo de los trabajadores a partir de diversas políticas de distribución del ingreso, habilitando asimismo una potente recomposición del tejido de PyMEs, las cuales ocupan un rol central en materia de empleo. Dentro de las políticas distributivas se destacaron el aumento del salario mínimo real, la multiplicación de los convenios colectivos de trabajo, ampliación y mejoras en las jubilaciones pasando de una cobertura del 66\% hasta alcanzar casi su universalización y distintas políticas sociales de diverso alcance, llevando a que en el conjunto del período kirchnerista el desempleo pasara del 20,4\% en el primer trimestre de 2003 al 5,9\% en el último de 2015, y los índices de pobreza e indigencia lo hicieran del 49,7\% y el $22,8 \%$ en 2003 al 19,7\% y al 4,4\% en $2015^{2}$, respectivamente. En este camino, el Estado amplió su capacidad de intervención económica, incluyendo la re-estatización de empresas en diversos sectores, introduciendo crecientes regulaciones de modo de orientar recursos a la sustentación del neo-desarrollismo naciente y al pacto populista en el cual basaba su lógica hegemónica.

Es en este punto donde las lógicas políticas, económicas e ideológicas se articulan definiendo efectos que se expresan tanto en el plano de la acción estatal interna como en la política exterior. Según Poulantzas (1981), el Estado es, por una parte, una condensación de relaciones de fuerzas, lo cual para el caso del kirchnerismo se expresó en los cambios al interior del bloque de poder, con una subordinación relativa de las fracciones de empresas de servicios privatizadas y del capital financiero respecto a un avance del capital productivo y exportador. Por otra parte, el Estado sin dejar de representar de manera predominante los intereses de la clase o fracción hegemónica, reviste una autonomía relativa, en tanto asegura el interés político general del conjunto del bloque en el poder organizando el "equilibrio inestable de compromisos" (Gramsci) entre las distintas fracciones dominantes al tiempo que establece esta hegemonía igualmente respecto de las clases subalternas. De este modo, entendemos que la lógica autonómica aparece simultáneamente, en el plano interno como vía de construcción del pacto populista y como condición de posibilidad de un proyecto que desde la periferia del orden global antagoniza , a nivel internacional, con algunas tendencias nodales del capitalismo de cuño neoliberal imperante. Así, la autonomía es clave tanto para la construcción política y económica nacional como para la implementación de un régimen de acumulación que trasciende las fronteras nacionales y busca aportar a la conformación de un orden posneoliberal.

La dinámica autonómica fue recuperada por diversos autores para el análisis de la política exterior (Simonoff 2009, 2013; Morasso, 2016; Busso, 2016, entre otros), y definida en el discurso oficial ya desde un primer momento por NK en frases como "Pensamos al mundo en argentino, desde un modelo propio" o "no deben esperarse de nosotros alineamientos automáticos" (25/3/2003). Asimismo, es necesario destacar, como puede leerse en Russel y Tokatlian (2002) así como en Morasso (2016), que en el contexto actual la autonomía es entendida como regionalización, por lo que la integración y unidad regional aparece como uno de los pilares de la acción externa durante el kirchnerismo, vista como condición necesaria para la ampliación de los márgenes de libertad también en el plano interno. Esta lectura llegará luego a su expresión más acabada en el discurso de CFK cuando afirma que: "la independencia y el

\footnotetext{
${ }^{1}$ En la década 2003-2013 se amplió la cantidad de parques industriales de 80 a 315, conllevando un aumento del $277 \%$ en las empresas industriales involucradas y del $241 \%$ de los trabajadores industriales, según datos del Ministerio de Industria.

2 Datos desocupación según INDEC del 2017 y datos de pobreza e indigencia de CIFRA (2015). Es conveniente aclarar que a partir de 2007 el gobierno nacional generó una intervención en el INDEC que generó una pérdida de confianza en los indicadores, particularmente vinculados al índice de precios, por lo que en dicha materia se tornó necesario recurrir a fuentes alternativas.
} 
crecimiento de nuestra región está fuertemente atados al proceso de integración. Si para nosotros la integración antes fue un desafío, ahora es una necesidad y es una necesidad de carácter estratégico" (1/7/2008).

En esta línea, durante el gobierno de NK se tendió a subrayar la prioridad del "MERCOSUR como proceso de integración y como la más importante vía de inserción de la República Argentina en la comunidad internacional" (18/6/2003). La relevancia del MERCOSUR puede visualizarse en la múltiples funciones que se le asignan, ya que como sostiene Zelicovich, el mismo "aparecerá como vía para la inserción internacional, en términos fundamentalmente de comercio e inversiones; como sustento para la generación de autonomía; como herramienta para la expansión hacia la región; como estrategia en las negociaciones con terceros; y como herramienta para la realización del modelo de desarrollo propuesto" (2011:185). Esta centralidad que recuperó el MERCOSUR no estuvo exenta de tensiones, las cuales se vincularon a la existencia de asimetrías en su interior, tanto a nivel del peso de las distintas economías como de las balanzas comerciales entre sus miembros, para los cuales se buscaron reforzar el diálogo y los mecanismos institucionales de compensación.

Esta construcción de autonomía como regionalización tuvo un hito fundamental en el rechazo al ALCA en 2005 liderado por los presidentes de Venezuela, Brasil y Argentina y acompañado por el conjunto de los gobiernos del MERCOSUR. Desde la visión presidencial, ya NK advertía previamente los problemas que contenía el planteo del ALCA, en tanto en la Cumbre Extraordinaria de las Américas de 2004 sostenía: "Un acuerdo no puede ser un camino de una sola vía, de prosperidad en una sola dirección; un acuerdo que no se haga cargo ni resuelva las fuertes asimetrías existentes no hará más que profundizar la injusticia y el quiebre de nuestras economías. Un acuerdo no puede resultar de una imposición en base a las relativas posiciones de fuerza" (13/1/2004). Es entonces en base a una búsqueda de alterar esas relaciones de fuerzas internacionales donde la integración latinoamericana aparece como indispensable y el rechazo al ALCA se erige como condición de posibilidad, dando origen a un nuevo proceso de regionalismo autónomo en el marco de la guerra de posiciones que comenzaba a definir al continente.

Asimismo, el No al ALCA se funda en la contrastación de los regímenes de acumulación en disputa y la conformación identitaria que demarca al adversario en torno a las figuras diversas del neoliberalismo: "Esa uniformidad que pretendía lo que dio en llamarse el 'Consenso de Washington'... hoy existe evidencia empírica respecto del fracaso de esas teorías" (4/11/2005) sostenía NK en la IV Cumbre de las Américas de 2005 fundamentando el rechazo al acuerdo de libre comercio, mientras reclamaba a EEUU y los organismos financieros internacionales tomar responsabilidad por haber promovido dichas políticas.

Esta línea dio un nuevo avance en diciembre de 2005 con el pago por adelantado y fin del acuerdo crediticio con el FMI, al cual NK caracterizó como "un paso trascendental, que nos permitirá mirar sin imposiciones, con autonomía y tranquilidad" y que se basó en el rechazo de las demandas que buscaba imponer dicho organismo en la política interna. Así, el FMI se convirtió en uno de los ejes de la crítica oficial: "desnaturalizado como está en sus fines el Fondo Monetario Internacional ha actuado, respecto de nuestro país, como promotor y vehículo de políticas que provocaron pobreza y dolor en el pueblo argentino, de la mano de gobiernos que eran proclamados alumnos ejemplares del ajuste permanente" (15/12/2005).

El rechazo al ALCA y el fin del tratado y supervisión del FMI fue la coronación de un proceso de ampliación de los márgenes de autonomía conquistado a través de la salida del default de la deuda pública. Del total de los US\$ 81.800 millones que entraron en cesación de pagos en 2001 el canje de 2005 fue aceptado en un 76,15\%, de modo que US\$ 62.300 millones fueron cambiados por los nuevos títulos elegibles, reduciéndose a US\$ 35.300 millones, representando una quita del 43,4\% (que en algunos análisis supera el 60\% calculando intereses y punitorios). Asimismo, comenzó a reestructurarse la deuda, perdiendo peso su componente externo (frente al interno e intra-sector público) y bajó la denominación en divisas frente a la deuda en moneda local, ampliando márgenes de autonomía evidenciados también en la mejora 
de todos los indicadores de endeudamiento en relación al PBI, reservas y exportaciones, originando la política de desendeudamiento ${ }^{3}$.

Aquí nuevamente observamos un puente entre la política interna y externa, ya que el desendeudamiento y la ruptura con el FMI, que ocuparon un vasto espacio en el debate que Argentina llevó a los foros internacionales, se ligó también a la visión del proyecto y el régimen de acumulación a promover tanto local como internacionalmente. Esto se constata en que los gobiernos kirchneristas motivaron una pérdida de centralidad de las estrategias de valorización financiera, con avances en la regulación del movimiento de los flujos de capitales especulativos y de la tasa de interés (con tendencia a ser negativa en términos reales), y promovieron una mayor gravitación de los conglomerados financieros públicos. Esta política se profundizó luego en los gobiernos de CFK con la re-estatización del sistema de jubilaciones y pensiones en 2008 (previamente en manos del capital financiero) y la reforma de la Carta Orgánica del Banco Central en 2012 con sus efectos de mayor regulación pública sobre el sector. Asimismo, la pérdida de peso de la deuda en el presupuesto nacional permitió amplificar las políticas distributivas avanzando con el precepto fundacional de "No se puede volver a pagar deuda a costa del hambre y la exclusión de los argentinos" (25/5/2003) y generando un cronograma de pagos compatible con la recuperación económica del país, que NK sintetizó en su alocución de "Nunca se supo de nadie que pudiera cobrar deuda alguna de los que están muertos" (25/9/2003) sostenida en su primera intervención en la Asamblea General de la ONU.

La estrategia definida para ganar márgenes de autonomía, en un contexto de relaciones de fuerzas internacionales aun definido por la unipolaridad, fue el multilateralismo y la conformación de contra-pesos tendiente a la multipolaridad.

NK planteaba en los foros internacionales que "no existe alternativa aceptable a la acción multilateral. Sólo el debate colectivo y el consenso de una mayoría de países pueden asegurar una acción genuina" (21/9/2004). De este modo, el multilateralismo, junto con el diálogo, aparecían como el único camino para la resolución de controversias.

Y en este sentido fue remarcado, tanto en sus discursos como luego en los de CFK, para remitir al reclamo de soberanía por Malvinas y las Islas del Atlántico Sur, entendidas como un enclave colonial sostenido por una potencia que hacía uso y abuso de su posición de miembro permanente del Consejo de Seguridad de la ONU y su capacidad de ejercer veto, desoyendo los numerosos reclamos de los organismos multilaterales de sentarse a dialogar con Argentina para dirimir el conflicto. También en este camino cobró fuerza la opción multipolar, en tanto las potencias aliadas al eje unipolar angloamericano no acompañaban el reclamo soberano argentino, la solidaridad se fue extendiendo con las potencias emergentes como China y Rusia, así como gran parte de los países de América Latina y África.

Sin embargo, no hubo un despliegue lineal de estas estrategias, ya que se conjugaron de forma particular en otros casos como las Misiones de Paz de la ONU. Si bien NK atribuía una "importancia significativa a la participación, junto con otros países de la región, en la Misión de Estabilización de las Naciones Unidas en Haití (MINUSTAH)" (20/9/2006) y la fundamentó como una acción de ayuda a un país necesitado, esta intervención también ha sido leída como una concesión funcional a Washington que validó el desplazamiento del presidente haitiano impulsado por EEUU (Aranda, 2004) y permitió evitar el uso de tropas norteamericanas allí para que estén disponibles en otras zonas del mundo, compensando, por ejemplo, la negativa de Argentina a enviar tropas a Irak, basada justamente en la unilateralidad de la acción.

\footnotetext{
${ }^{3}$ El peso de la deuda sobre PBI disminuyó significativamente, pasando de representar el $127 \%$ del PBI en 2004 al 74\% en 2005, cayendo al 56\% en 2007. También la relación deuda pública externa sobre reservas internacionales mejoró: del pico más alto de 2002 en $823 \%$ se redujo al $135 \%$ en 2007. Otra mejoría considerable se observa en la relación deuda/exportaciones, de $281 \%$ en 2004, cayó con el canje a $131 \%$ en 2005 terminando en $94 \%$ en 2007. Todos estos indicadores mejorarían aún más en los gobiernos de CFK.
} 
Otro tipo de concesión en política interna a las demandas internacionales de Washington puede ser encontrada en la sanción de las leyes antiterroristas en Argentina. Aquí se da la particularidad de que Argentina mantuvo, a tono con EEUU, un discurso que puso en primer lugar la lucha contra el terrorismo, recordando los casos de los atentados contra la Embajada de Israel en 1992 y contra la AMIA en 1994, asemejando dichos atentados al sufrido por EEUU en 2001 contra las Torres Gemelas. Sin embargo, en contraposición con Washington, la política exterior argentina esgrimió, como aspecto inescindible para la seguridad global, la defensa irrestricta de los DDHH, criticando de forma creciente el guerrerismo norteamericano. En materia de DDHH, Argentina promovió, junto a Francia en la ONU, la sanción de la Convención Internacional para la Protección de Todas las Personas contra las Desapariciones Forzadas, que define a la desaparición como crimen de lesa humanidad y funda el Comité contra la Desaparición Forzada para seguir dicha problemática. Además, en el ámbito de la Organización de Estados Americanos (OEA) se aprobó por consenso la resolución sobre el Derecho a la Verdad presentada por Argentina, implicando un aporte al Sistema Interamericano de Protección y Promoción de los Derechos Humanos. Sin embargo, la sanción de las leyes antiterroristas, que podrían alinearse con el precepto de lucha contra el terrorismo constitutivo de la política exterior, fue leída por numerosas organizaciones sociales al interior del país como una concesión a EEUU que podía traer consecuencias negativas si futuros gobiernos la utilizaran contra la lucha popular como sucedía en otros países de la región.

Más allá de estas ambigüedades, la búsqueda de contra-pesos multipolares se vio expresada en el avance tanto de la integración regional como de la cooperación Sur-Sur. Según Lechini y Giaccaglia (2016), esta cooperación tuvo como actores predominantes a las potencias emergentes aportando al rediseño de la arquitectura de poder mundial procurando una nueva relación entre economía y política. "En el caso de Argentina, la idea de cooperación Sur-Sur reapareció en la política exterior argentina durante la administración de Néstor Kirchner, cuya conceptualización se reinsertó en el discurso oficial y se impulsaron algunas iniciativas tanto multilaterales como bilaterales con países latinoamericanos y extra-regionales" (Lechini y Giaccaglia, 2016:8), aprovechando al MERCOSUR como plataforma de relacionamiento, a través de la cual Argentina frecuentemente acompañó las propuestas Sur-Sur impulsadas principalmente por Brasil en su creciente protagonismo global.

\section{Los gobiernos de CFK: discurso, proyecto e integración}

\subsection{Pueblo, nación y neo-desarrollismo en tiempos de crisis global}

La llegada de CFK al gobierno implicó la continuidad y consolidación de los factores centrales de la estrategia de inserción internacional. En su discurso de asunción CFK reforzó las bases ideológicas del proyecto oficial señalando que "uno puede encontrar en la defensa irrestricta de sus propios intereses, como Estados y sociedades, la clave de ese avance, la clave de ese desarrollo. Por eso, pueblo y nación en tiempos de globalización siguen más vigentes que nunca, representar los intereses de los argentinos" (10/12/2007).

Aquí parece necesario recuperar la conceptualización que realizara O’Donnell (1978) de estos términos constitutivos de la identidad nacional-popular. Dicho autor indaga cómo en las sociedades capitalistas el vínculo entre Estado y sociedad se encuentra fracturado por las relaciones de dominación y explotación que las atraviesan, por lo cual se postulan diversas mediaciones para reengarzar ese vínculo ignorando los clivajes de clase. Estas mediaciones constituyen entonces instancias aglomeradoras, puestas sobre el fraccionamiento de la sociedad. La restitución de lo nacional-popular, característica del período bajo estudio, presenta a la nación como arco de solidaridades que constituye un "nosotros" definido por la común pertenencia a un territorio estatal, como referente homogeneizante y general. Y lo liga al pueblo o lo popular, que emerge porque la postulación indiferenciada del interés general de la nación no alcanza a tapar las evidencias de desigualdad en la sociedad burguesa. Mientras, bajo el régimen neoliberal, esa desigualdad se expandió junto con el deterioro de las condiciones de 
vida de las clases subalternas, el kirchnerismo postulaba un Estado que debía actuar en un sentido equiparador. Así, la recuperación de lo nacional-popular actuaba entonces como referente y fundamento de dicha acción estatal desplegando un proyecto que expresaba (en términos gramscianos) una unidad de fines políticos y económicos que impulsarían al máximo desarrollo al conjunto de las "energías" nacionales. Pero estas energías trascendían de escala para encontrarse como estandartes que pervivían frente al avance de la globalización, por lo que las categorías de pueblo y nación mantenían su validez para pensar la acción estatal externa. La autonomía como regionalización implicaba que la realización de los intereses del pueblo y la nación en Argentina superara esta escala para percibirse como parte de un pueblo y una nación mayor, avanzando hacia una escala latinoamericana: un "país latinoamericanista" como dijera NK (1/3/2007).

Esto se vincula, por otra parte, con que la matriz populista en términos ideológicos se materializa en política pública en el despliegue del neo-desarrollismo, presentado por CFK como "un modelo económico de acumulación con matriz diversificada e inclusión social" (10/12/2007). El régimen neo-desarrollista con su componente de inclusión social se constituye entonces en una propuesta político-económica desarrollada no sólo nacionalmente sino factible de ser internacionalizada. Debemos notar que en este contexto, Brasil, la potencia regionalglobal, venía promoviendo un régimen de similares características, lo cual daba mayor sustancia a este posicionamiento ${ }^{4}$.

Fue particularmente en el contexto de irrupción de la crisis internacional, que actuó de corolario externo de la crisis interna desatada tras la derrota del kirchnerismo en el conflicto agrario de 2008, donde la caracterización y disputa de proyectos dio un salto cualitativo. Con el avance de los condicionamientos externos, CFK esgrimió en la ONU una lectura de la situación internacional ligada a la distinción de dos modelos en disputa, con los actores que los sustentan:

\begin{abstract}
No va a venir ninguna calificadora de riesgos, tampoco va a venir el Fondo Monetario Internacional a decir lo que tiene que hacer este gran país que ha crecido históricamente a raíz de la economía real, y que realmente hoy tiene problemas a partir de una economía de casino o de ficción, donde se ha creído que el capitalismo solamente puede producir dinero. Yo siempre digo que el capitalismo, señoras y señores, ha sido imaginado para ganar dinero, pero a partir de la producción de bienes, de servicios y de conocimientos, el dinero solo no produce más dinero, necesariamente tiene que pasar por el circuito de la producción, del trabajo, del conocimiento, de los servicios, de los bienes, para que entonces en un círculo virtuoso pueda además generar bienestar a toda la sociedad (23/9/2008).
\end{abstract}

En la apertura de las sesiones ordinarias del Congreso argentino en 2009, CFK planteó una lectura de la crisis mundial como caída del "fundamentalismo de mercado" impulsado por los organismos financieros internacionales, y como crisis de una forma de relacionarse a nivel internacional basada en la subordinación y no en la cooperación (1/3/2009). La crítica al FMI se robusteció en el marco de una crítica a lo que luego, en la Cumbre del G20 en Cannes, denominaría como un capitalismo anarco-financiero (3/9/2011). Esta conceptualización se liga estrechamente con los factores que el kirchnerismo identifica como causantes de la crisis global: "la famosa crisis de las sub prime, hoy ha terminado en algo más que una crisis de pobres que no puede pagar sus hipotecas, se ha corrido finalmente el velo y se ha descubierto, o por lo menos ha quedado expuesto a la luz pública, que ha sido precisamente la administración financiera de capitales sin ningún tipo de regulación, no ya en beneficio de los sectores más empobrecidos sino de los sectores más ricos de la sociedad, la causante de esto que ya es una crisis global" (25/9/2012). En este sentido, el kirchnerismo se opone a las recetas promovidas por el FMI basadas en el ajuste y el salvataje al capital financiero, proponiendo una apuesta por

\footnotetext{
${ }^{4}$ Cabe aclarar que el caso de Brasil presentó la particularidad de sostener inicialmente un mayor nivel de continuidad con el régimen neoliberal, particularmente en la estrategia macroeconómica durante el primer gobierno de Lula Da Silva, y que luego las transformaciones de carácter neo-desarrollista fueron avanzando con mayor énfasis durante el segundo gobierno de Lula y los gobiernos de Dilma Rousseff (Morais y Saad-Filho, 2011; Schutte, 2013).
} 
los mercados internos basada en la producción y el empleo, compatible con una ampliación del comercio internacional.

Aquí vemos otro caso donde la acción externa e interna converge mostrando una unidad de proyecto. En la escala nacional, el gobierno de CFK desplegó un ambicioso plan anti-crisis basado en principios opuestos a los de la ortodoxia neoliberal. Mientras el FMI proponía inyectar recursos al capital financiero, en Argentina la base del plan partió de la estatización de las Administradoras de Fondos de Jubilaciones y Pensiones (AFJP), que estaban en manos de los conglomerados financieros. Contra la desregulación y liberalización económica se desplegaron mayores herramientas de control del comercio exterior, aplicando nuevas medidas sobre las importaciones para proteger la producción local. Lejos de ajustar el gasto público, el Estado se colocó como motor de la demanda agregada, por ejemplo, otorgando créditos para incentivar el consumo, cuadruplicando el Programa de Recuperación Productiva para asistir a las empresas en crisis a condición de que no echaran trabajadores, estableciendo un masivo plan de obra pública y políticas favorables a las clases subalternas como el incremento del salario mínimo, la creación de mil cooperativas y, la más destacada, la Asignación Universal por Hijo. De hecho, el plan mostró buenos resultados llevando a un fuerte crecimiento en 2010 y $2011^{5}$.

Aun así, la doble lógica hegemónica del populismo estaba atravesada por distintos desafíos. Por un lado, el pacto populista comenzaba a crujir con la defección de crecientes sectores de las clases dominantes y su pasaje a la oposición. Esto llevó a la dinamización de la confrontación con grupos concentrados del bloque de poder al tiempo que habilitó la articulación de mayores demandas de las clases subalternas en lo que caracterizamos como un proceso de radicalización progresista ${ }^{6}$, donde las mediaciones pueblo y nación adquirían un nuevo volumen con el avance del proceso de inclusión social y el fortalecimiento del Estado. En este camino, ubicamos a la estatización y emisión gratuita de la televisación del fútbol, la Ley de Medios con su perfil anti-monopólico, la expansión del Estado con las acciones adquiridas con la estatización de las AFJP y las nuevas estatizaciones como la de Aerolíneas, la Fábrica Militar de Aviones, entre otras, sumando luego un hito de soberanía económica y tecnológica con el lanzamiento de los satélites geo-estacionarios Arsat-1 y Arsat-2 de producción estatal. También encontramos políticas de transferencia directa hacia las clases subalternas, como las de ingresos y las políticas sociales, e indirecta, como la multiplicación de presupuesto en educación, ciencia y tecnología, los avances en salud, como la duplicación del calendario de vacunas, los planes de salud reproductiva, mental, entre otros. Asimismo, se conquistaron nuevos derechos civiles como el matrimonio igualitario y la Ley de Identidad de Género, en una batalla política y cultural que enfrentaba a los sectores más conservadores del país.

Esta base de políticas públicas, por un lado, posibilitaba afirmar el proyecto hegemónico, dotando al neo-desarrollismo argentino de legitimidad e impulsaba al kirchnerismo a alcanzar tres gobiernos consecutivos, pero también agudizaba la disputa antagónica con grupos poderosos tanto a nivel nacional como internacional.

\subsection{El avance de la integración y la contra-ofensiva imperialista}

El MERCOSUR, durante los años de CFK, estuvo marcado por tensiones que expresaban en una doble tendencia. Por un lado, estuvo atravesado por distintas rispideces como el disímil posicionamiento de Argentina y Brasil en la OMC en materia arancelaria y las nuevas medidas proteccionistas que ambos países desplegaron en el marco de la crisis internacional, y los descontentos persistentes en miembros como Uruguay reabriendo el debate por la búsqueda de establecer tratados de libre comercio con terceros países, entre otros factores. Por otro lado, la posibilidad de redoblar la apuesta del MERCOSUR a través de su ampliación, con Argentina promoviendo la incorporación de aliados claves en el bloque popular latinoamericano como

\footnotetext{
${ }^{5}$ Se calcularon aumentos del PBI del 9,2\% por ciento en 2010 y 8,9\% en 2011 (datos año base 1993).

${ }^{6}$ Para ver en profundidad el análisis de estos fenómenos ir a Varesi (2011).
} 
Venezuela y Bolivia, que tampoco estaba exenta de resistencias como las que el parlamento de Paraguay ponía frente a Venezuela. Aun así, este proceso de integración sería complementado por nuevas experiencias que habilitaron un fortalecimiento del regionalismo autónomo.

La regionalización conquistó un nuevo hito con el lanzamiento de la Unión de Naciones Suramericanas (UNASUR). En UNASUR el Estado cobraba un rol central, y se valoraba la presencia de las empresas estatales en los distintos proyectos de cooperación e integración tanto en infraestructura como en energía. En materia económica, para Bernal-Meza (2013) la importancia de UNASUR estuvo puesta más en el desarrollo de infraestructura que en el comercio, en tanto se mostraba flexible para aceptar en su interior las distintas alternativas arancelarias. Se tomó el plan Iniciativa para la Integración de la Infraestructura Regional Suramericana (IIRSA), creado en el año 2000 durante la primera Cumbre Sudamericana como una forma de facilitar y promover la integración regional en materia de infraestructura, con el fin de eliminar las barreras físicas entre los países de la región, fomentar el comercio y la formación de cadenas productivas en la región e incluye proyectos de transporte, energía, comunicaciones y la creación de una red de gasoductos. Así, en el marco de la Tercera Reunión del Consejo de Jefas y Jefes de Estado de la UNASUR, el 28 de enero de 2009 en Quito, IIRSA fue incorporado en el Consejo Suramericano de Infraestructura y Planeamiento (COSIPLAN), pasando a ser uno de los doce Consejos Ministeriales y Sectoriales de UNASUR. Brasil se fue conformando desde un comienzo como el principal impulsor e inversor de los planes de infraestructura en tanto los comprende como un paso clave para su propia consolidación como potencia económica global. Asimismo, estos planes han sido cuestionados por sus correlatos ambientales y la depredación no sustentable de los recursos naturales que, según sus críticos, involucran. También cabe resaltar que la diversidad de proyectos económicos y de inserción al comercio exterior de los países miembros, dificultó poder constituir a UNASUR en proceso de integración de carácter integral para la región sudamericana.

Aun así, UNASUR adquirió una potente relevancia geopolítica a partir de dos aspectos interdependientes: su agenda de seguridad y la resolución de conflictos.

En la Cumbre de UNASUR de 2010, CFK identificó diversas estrategias desplegadas por las economías centrales de "exportación de la crisis" a los países emergentes así como movimientos tendientes a la desestabilización de la región, como el caso de la instalación de bases militares norteamericanas en Colombia. Frente a esto, el kirchnerismo volvió a postular la necesidad de unidad latinoamericana como vía de fortalecimiento para enfrentar los desafíos globales junto a la construcción de una nueva institucionalidad regional para "preservar a nuestra América del Sur como una región de paz, una región donde las doctrinas de unilateralidad no vengan precisamente a perturbar" esa paz (28/8/2009), abordando y procesando al interior de la región las situaciones conflictivas sin injerencias de las potencias externas. Una vez más la autonomía aparecía como regionalización, en tanto que el par multilateralismo y cooperación se enfrentaba a la opción de unilateralismo y subordinación aplicada por las potencias occidentales. En este camino, cabe señalar que durante los gobiernos de CFK, UNASUR fue cobrando mayor gravitación en el discurso oficial, incluso por sobre el MERCOSUR, mostrándose como espacio privilegiado de cooperación e integración.

De hecho, según Bernal-Meza (2013), al incorporar una agenda de seguridad autónoma de EEUU, UNASUR se constituyó en un desafío al orden norteamericano en el hemisferio occidental, el cual incluye al Tratado Interamericano de Asistencia Recíproca (TIAR) ${ }^{7}$. Este tratado había sido firmado en 1947, en consonancia con la Doctrina Monroe (1823) que afirmaba que ninguna potencia extra continental debía tener injerencia en América dejando el rol de gendarme a los EEUU, bajo el lema "América para los americanos" (donde América es

\footnotetext{
${ }^{7}$ Según el TIAR "un ataque armado por cualquier Estado contra un Estado Americano, será considerado como un ataque contra todos los Estados Americanos, y en consecuencia, cada una de las Partes Contratantes se compromete a ayudar a hacer frente al ataque en ejercicio del derecho inmanente de legítima defensa individual o colectiva que reconoce el Artículo 51 de la Carta de las Naciones Unidas".
} 
todo el continente pero los "americanos" terminan siendo sólo los de EEUU). En ese sentido, la OEA (fundada en 1948) también cumplía su rol de agencia de seguridad regida usualmente bajo la influencia de EEUU, en cuyo país tiene sede, y a partir de cuya decisión, por ejemplo, se excluyó a Cuba desde 1962 hasta 2009. Debe recordarse que tanto el TIAR como la OEA habían abandonado, contra sus propias cláusulas, a la Argentina en la guerra de Malvinas.

El Consejo de Defensa Suramericano (CDS) de UNASUR, comenzó a formarse entonces como una alternativa para la resolución sudamericana de los conflictos sudamericanos. Así, el CDS ratificaba la autodeterminación de los pueblos, la plena vigencia de las instituciones democráticas y la protección de los Estados frente a amenazas internas o externas, junto al respeto de los $\mathrm{DDHH}$, la defensa de los recursos naturales y la promoción de medidas de confianza y transparencia en asuntos militares y de defensa (Bernal-Meza, 2013). Asimismo, el CDS promovía la conformación de una industria sudamericana de defensa así como la formación propia de los cuadros de altos oficiales, restando influencia a EEUU. La capacidad política de UNASUR para resolver conflictos desde la propia región se hizo notoria en casos como los intentos de desestabilización y secesión en Bolivia, el conflicto político entre Venezuela y Colombia, el ataque de Colombia en territorio ecuatoriano a las FARC, el intento de golpe en Ecuador, entre otros.

Otro avance en materia de integración se dio con la creación de la Comunidad de Estados Latinoamericanos y Caribeños (CELAC) realizada en Caracas, República Bolivariana de Venezuela, en el marco de la III Cumbre de América Latina y el Caribe sobre Integración y Desarrollo (CALC) y de la XXII Cumbre del Grupo de Río, reunidas los días 2 y 3 de diciembre de 2011. Con la firma de la Declaración de Caracas nació la CELAC delineando diversos temas a abordar, como la necesidad de construir una nueva arquitectura financiera, demanda sostenida también por la política exterior argentina en todos los foros internacionales. La articulación de todos los países latinoamericanos, incluyendo a Cuba, y la exclusión de Canadá y EEUU, fue anotada como un nuevo desafío al poder de este último.

Nos encontramos así en un escenario contradictorio: los principales avances en materia de integración regional como el lanzamiento de UNASUR y CELAC se dieron un contexto donde EEUU recuperaba la iniciativa sobre la región y lanzaba su contra-ofensiva. La misma combinó aspectos políticos, económicos, ideológicos y militares y puede ser sintetizada en dos aristas: la desestabilización con estrategia de "golpe suave" y la asfixia económica de los rivales.

La contra-ofensiva comenzó a sustanciarse con el lanzamiento en 2008 de la IV Flota norteamericana para controlar la región y tuvo su primer éxito en el golpe de Estado en Honduras en 2009. Debe remarcarse que nos encontramos ante un neo golpismo el cual está encabezado más abiertamente por civiles, con el apoyo tácito o explícito de las fuerzas armadas, utiliza una violencia menos ostensible e intenta preservar una semblanza institucional mínima (Tokatlian, 2009). El segundo caso en la región fue la destitución del presidente Lugo en Paraguay, lo cual motivó la suspensión de dicho país del MERCOSUR y brindó el contexto para la incorporación de Venezuela al mismo, mostrando un genuino tironeo de relaciones de fuerzas entre el bloque popular latinoamericano y EEUU y sus aliados criollos en la guerra de posiciones continental. Al respecto, la propia CFK denunció el intervencionismo norteamericano y el rol de los golpes suaves para dirimir la disputa de proyectos, en la VII Cumbre de las Américas:

Han surgido nuevas formas más sutiles de intervención e influencia en nuestros gobiernos a través de lo que se conoce como "los golpes suaves". Golpes suaves donde se utilizan medios masivos de comunicación multinacionales, denuncias falsas, asociaciones caprichosas de Estados con otros Estados para hacer no sé qué cosas y qué conspiraciones. Son más sutiles, son más sofisticadas, pero no por ello dejan de ser intervenciones y siempre encuentran su origen en nuevas organizaciones bajo el nombre de ONGs. ... ¿por qué se combate y se tilda de populistas precisamente a los gobiernos que en América del Sur, que en la América Latina, han sido los que mayores logros en materia de derechos humanos, de equidad, de inclusión, de educación, de salud 
han logrado? ¿Por qué apoyaban o apoyan a gobiernos que plantean políticas neoliberales que excluyen ciudadanos? (11/4/2015).

La otra arista de la contra-ofensiva fue la asfixia económica, ya sea de carácter "molecular" como la larga guerra económica contra Venezuela o con estrategias contra sus ingresos básicos como en el desplome del precio del petróleo. En referencia a la Argentina tuvo un hito particular: el asedio de los "fondos buitres".

Tras la recuperación económica argentina de 2010 y 2011, el segundo gobierno de CFK comenzaba a enfrentar distintos signos de desgaste a nivel del régimen neo-desarrollista tales como la crisis energética (la cual motivó la estatización de YPF que fue la más importante de todo el período), cierto estancamiento de la industria, una inflación persistente y la reaparición de la restricción externa (Varesi, 2018). Para enfrentar este último problema, el gobierno de CFK buscó retornar a los mercados financieros internacionales. Tras realizar un segundo canje de deuda exitoso en 2010, concretar las negociaciones para pagar la deuda con el Club de París en 2014 y arreglar con las empresas que tenían juicios en el CIADI contra Argentina, el retorno a los mercados financieros internacionales fue bloqueado a partir del fallo del juez Griesa de Nueva York a favor de los "fondos buitres", el 7,3\% que había rechazado los canjes y que buscaba cobrar muy por encima de lo pautado con el $92,7 \%$ de los acreedores.

La contra-ofensiva fue leída y denunciada por CFK como un castigo por ser parte de quiénes venían desafiando al orden del capitalismo financiero mundial: “¡El problema es que no hemos vuelto a pedir prestado, que no hemos vuelto a hacer negocios financieros! ¡Ese es el problema! ¡Nos quieren volver a endeudar! (...) hemos ido a contramano de todas y cada una de las cosas que nos decían que teníamos que hacer y nos fue bien. Eso es lo que no nos perdonan, y por eso nos quieren castigar" (1/3/2013).

Asimismo, CFK entendía que el tema de los fondos buitres no era un asunto aislado sino que existía complicidad de EEUU, como lo declamó en la ONU: "Argentina con la complicidad del sistema judicial de este país está siendo acosada por estos fondos buitres (...) se le ha reconocido a ese 1 por ciento en un juicio aquí, en la jurisdicción de Nueva York, ha sido una tasa de 1.608 por ciento, en cinco años, en dólares (...) hoy están obstruyendo el cobro de quienes confiaron en la Argentina, de ese 92,4 por ciento" (24/9/2014). En dicho foro CFK terminó de describir la acción de los fondos buitres y su poder desestabilizador caracterizándolos como terroristas económicos: "Fondos buitres que además amenazan y hostigan con acciones sobre la economía de nuestro país, provocando rumores, infamias y calumnias desde lo personal hasta lo económico y financiero, de modo tal de actuar como verdaderos desestabilizadores de la economía, casi una suerte de terrorismo económico y financiero" (24/9/2014). En dicho conflicto, con la resolución de Griesa firme y la decisión de la Corte Suprema norteamericana de no tomar el caso convalidando tácitamente el fallo, y con los fondos de los acreedores del canje inmovilizados, las calificadoras de riesgo dieron el golpe final sentenciando la situación como un default técnico, liquidando la estrategia oficial para volver a acceder a los mercados internacionales de crédito.

Esto motivó la última saga de protagonismo del kirchnerismo en política exterior. Argentina tomó la iniciativa y, contra la voluntad de EEUU y sus aliados, llevó a la ONU la propuesta de Resolución sobre Principios Básicos para la Reestructuración de Deudas Soberanas, tras haberla consensuado con el G77+China. Esta resolución fue aprobada en Naciones Unidas en 2015 con 136 votos positivos. La misma implicaba el reconocimiento de la soberanía de los Estados para formular sus políticas macroeconómicas y, por lo tanto, para poder reestructurar sus deudas; además establecía la inmunidad soberana para evitar embargos a los Estados por fallos en torno a endeudamientos y asentaba que las condiciones de canje de deuda aceptadas por la mayoría debían ser acatadas por el resto, para prevenir futuras situaciones como que la que Argentina estaba padeciendo con los fondos buitres.

En un contexto de recrudecimiento de la confrontación del bloque popular latinoamericano con EEUU y sus aliados, Argentina siguió demandando la democratización de 
los organismos multilaterales, denunciando al Reino Unido y su ocupación colonial de Malvinas, criticando las políticas de ajuste promovidas por el FMI y señalando la complicidad de las potencias occidentales en el financiamiento al terrorismo que ellas mismas decían combatir, como sucedió en el caso de Siria, donde según CFK quienes eran caracterizados como "freedom fighters" por alzarse en armas contra el gobierno de Bashar al-Ássad, aliado de Rusia, luego terminaron siendo parte del ISIS.

Justamente en este avance de la confrontación, Argentina reforzó su apuesta por la multipolaridad global buscando generar un contra-peso a través del acercamiento al eje de los BRICS y, en particular, a las potencias emergentes como Rusia y China. Con estos últimos se firmaron acuerdos estratégicos en materia de colaboración económica, promoviendo obras de infraestructura, ampliando el comercio bilateral y estableciendo mecanismos de asistencia financiera como los swaps de monedas con China, así como acuerdos en materia de ciencia, comunicación y apoyo mutuo en los organismos internacionales buscando posicionamientos comunes.

Sin embargo, los condicionamientos internos y externos, visibles por ejemplo en el caso AMIA con el Memorándum de entendimiento con Irán convertido en un elemento de disputa no sólo nacional sino internacional y agravado luego por la muerte del fiscal de la causa, Alberto Nisman, el bloqueo de acceso a los mercados financieros internacionales ya mencionado, las crecientes rispideces con las potencias occidentales y el deterioro del contexto político y económico nacional, comenzaron a marcar un nuevo estado de relaciones de fuerzas. El neodesarrollismo argentino llegaba a 2015 con un creciente deterioro de las cuentas fiscales y un primer año de déficit comercial, dificultades para sostener el crecimiento económico, un proceso inflacionario persistente y un cuadro de restricción externa importante, en el marco del colapso del pacto social con el enfrentamiento de los principales grupos del gran capital local y transnacional, incluyendo a los grandes medios de comunicación con su potencia ideológica y cultural, y con dificultades para realizar un salto cualitativo basado en el poder de las clases subalternas que le permitiera resistir los embates surgidos desde el interior de las clases dominantes. Así, el kirchnerismo como experiencia nacional-popular local y sus aliados que participaban del "giro a la izquierda" regional, perdían terreno frente a la contra-ofensiva imperialista y los nuevos-viejos vientos de reformas neoliberales y neoconservadoras que terminarían de sellar la suerte en Argentina en 2015, avanzando también sobre gran parte de América Latina.

\section{Conclusiones}

En la primera década y media del siglo XXI, nuestro continente se encontró atravesado por una guerra de posiciones librada entre el bloque del imperialismo norteamericano y sus aliados criollos, y los movimientos populares y las fuerzas políticas de izquierda y progresistas que, accediendo a distintos gobiernos de la región, conformaron el bloque popular latinoamericano. Es en este último bloque, habitado por proyectos heterogéneos de tipo socialista y neo-desarrollista, unidos por la crítica al régimen neoliberal, la búsqueda de mayores niveles de autonomía con fortalecimiento de los Estados y el despliegue de políticas favorables a las clases subalternas, donde podemos ubicar la experiencia kirchnerista.

En este contexto donde la unipolaridad global y las políticas del Consenso de Washington presentaban fuerte desgaste, emergió el kirchnerismo a partir de su acceso al gobierno en 2003. Su conformación como fuerza político-hegemónica se dio al ritmo de una estrategia que caracterizamos en términos de la doble lógica del populismo. Por un lado, gestando una articulación de demandas, particularmente aquellas puestas en escena durante la crisis de 2001, construyendo su propia identidad al calor del antagonismo que ubicaba a las figuras diversas del neoliberalismo como adversario. Por otro lado, desplegando el populismo como pacto social, buscaba articular intereses de fracciones de clases dominantes y subalternas, dirigidos desde el Estado en la formación de un proyecto político-económico que construyó el 
neo-desarrollismo en Argentina. Así, la identidad de la fuerza en ascenso hegemónico incluyó componentes del peronismo clásico, de la generación de los años 70, del progresismo democrático de los 80 y un perfil latinoamericanista ligado al contexto regional del "giro a la izquierda".

A partir de esta caracterización, analizamos distintos factores que constituyen un puente entre las escalas nacional, regional e internacional, configurando una unidad relativamente consistente de la acción estatal en dichas escalas a lo largo de todo de período de estudio. Así, en materia de política exterior, señalamos diversos aspectos comunes de los tres gobiernos kirchneristas.

En primer lugar, aparece la crítica al orden económico imperante y la propuesta de una alternativa. En este punto, indagamos, aspectos ligados al discurso, el cual instituye la base ideológica que inspira el componente de política pública, construyendo una lectura común del presente y trazando los rasgos centrales del proyecto a construir. Notamos que el neoliberalismo, luego denominado por CFK como "capitalismo anarco-financiero", aparece primero como el orden a superar y de cuya crisis emergen los distintos gobiernos del bloque popular de la región, y luego, como el orden que busca ser restablecido por el bloque norteamericano para ocluir dichos procesos populares. Así, la construcción de identidad en base a la confrontación con aquel adversario constituye una constante del período 2003-2015. Frente al capitalismo financiero se postula un capitalismo "nacional" o "productivo" que cobra diversas denominaciones, particularmente como modelo de crecimiento con inclusión social.

Los lineamientos claves del proyecto a construir se materializaron en distintas iniciativas de política pública que dieron forma al régimen neo-desarrollista. Éste tuvo como pilares el desarrollo productivo, con regulación de las tarifas de servicios y del sector financiero, la creación de empleo y las políticas sociales y de ingresos favorables a las clases subalternas, junto con un apoyo a la recomposición del tejido de pequeñas y medianas empresas. Este cuadro a su vez expresaba un cambio en las relaciones de fuerzas al interior del bloque de poder en consonancia con la nueva orientación de la acción estatal, que procuraba organizar el pacto social. Este tipo de estrategia implicaba la ampliación de la autonomía relativa del Estado para sustentar los equilibrios siempre inestables entre las fracciones dominantes y subalternas. Asimismo, las mediaciones pueblo y nación, vitales para esta estrategia hegemónica no se proyectaron sólo en el plano interno sino también en la escala regional.

Esto nos ubica en un segundo aspecto nodal: la ampliación de autonomía en el plano interno estaba dialécticamente vinculada a una ampliación de la autonomía en el plano externo. En este camino, visualizamos la potencia de la autonomía como regionalización, en tanto se identifica la integración regional como una condición indispensable para poder sustentar el proyecto a nivel nacional, de modo de lograr una inserción positiva en la globalización, e incluso para poner en debate características importantes de ésta. En este punto, se evidencia que el proyecto desplegado por el kirchnerismo no visualizaba un cierre de fronteras en términos nacionalistas (aunque sí promovía un mayor nivel de regulación estatal), sino que identificaba las asimetrías que caracterizan las relaciones de fuerzas internacionales y buscaba el fortalecimiento de la integración regional, primero MERCOSUR, luego UNASUR y CELAC, como vía para generar mejores condiciones de negociación con otros bloques.

De este modo, observamos un puente con dos vías. Una que va desde la autonomía relativa del Estado en el plano interno a la autonomía como regionalización en el plano internacional. Y otra que va desde la ampliación de la autonomía a partir de la integración regional, materializada con la conformación del bloque popular latinoamericano, para habilitar la sustentación del proyecto hegemónico a nivel local. Esta relación entre ambos planos implica, a su vez, la disputa de proyectos en ambas escalas: la búsqueda de internacionalización del neodesarrollismo en contraposición a la persistencia del régimen neoliberal, y la disputa de dichos proyectos en el plano nacional. 
La evidencia de este vínculo se observa en el camino que va del No al ALCA a la conformación del regionalismo autónomo en Latinoamérica, con el MERCOSUR como base neo-desarrollista, UNASUR como plataforma política del bloque popular en Sudamérica y la CELAC como la aspiración de unidad continental latinoamericana excluyendo a Norteamérica. Asimismo, la contra-ofensiva imperialista se materializó luego desde las acciones internacionales como la reactivación de la IV Flota, el despliegue de la estrategia neo-golpista del bloque de EEUU y sus aliados criollos (efectivizada en los casos de Honduras, Paraguay y, más adelante, Brasil), hasta las estrategias de asfixia y bloqueo económico como las promovidas contra Venezuela o contra la Argentina a través del caso de los fondos buitres, procurando la restauración neoliberal en cada uno de los países donde hizo pie.

Esto nos lleva a un tercer factor clave: la estrategia de multilateralismo y multipolaridad. Frente a un cuadro de relaciones de fuerzas internacionales tensionado entre la búsqueda de EEUU y sus aliados de la OTAN para restablecer la unipolaridad desgastada, incluyendo acciones unilaterales de apropiación de los recursos naturales y ocupación de posiciones estratégicas mediante la guerra, como en Medio Oriente, el multilateralismo orientado a construir las acciones internacionales por consenso, con legitimidad institucional y respeto a los DDHH, apareció como uno de los argumentos centrales de los gobiernos kirchneristas. Asimismo, la autonomía como regionalización encontraba como condición de sustentabilidad el avance hacia un mundo multipolar. De allí, que las alianzas estratégicas establecidas con China y Rusia en particular, y el acercamiento a los BRICS, en general, fueron visualizados como acciones relevantes en búsqueda de gestar contra-pesos que permitieran resistir la contra-ofensiva imperialista y sostener márgenes de autonomía para el desarrollo de los procesos populares.

Finalmente, vale la pena recordar que todo análisis en términos de relaciones de fuerzas implica, por un lado, una aspiración a percibir la articulación de múltiples dimensiones y escalas del objeto de estudio, y por otro, la certeza del movimiento y la búsqueda de comprensión de dicho dinamismo que descristaliza las relaciones de poder y las observa como proceso. Algunas preguntas que deja el análisis de relaciones de fuerzas internacionales, en su doble vía de las potencias y bloques en la política internacional y de los modos de producción y regímenes de acumulación que dirigen (y disputan) la economía global pueden ser: ¿cuáles fueron los alcances de las experiencias del bloque popular latinoamericano tanto en modificar el plano de la integración regional como en transformar las estructuras políticas, sociales y económicas de los países donde se desplegaron? ¿cuáles fueron sus límites? ¿qué estrategia deben darse los procesos que buscan gestar un nuevo orden global que supere la fase neoliberal de la globalización y constituya otro basado en el multilateralismo y la multipolaridad? ¿cómo pueden los países de América Latina quebrar la dependencia y emerger de la periferia global a un rol protagónico compatible con la mejora de las condiciones de vida de las mayorías populares?

\section{Referencias bibliográficas}

ACTIS, E., BUSSO, A., CALDERÓN E. y ZELICOVICH, J. (2017). De la permisibilidad a la restricción. Un análisis de la incidencia de los condicionantes sistémicos sobre la política exterior del kirchnerismo (2003-2015), en Cuadernos de Política Exterior Argentina (Nueva Época), 125, enero-junio, CERIR, Rosario, pp. 48-70

ARANDA, R. A. (2004). La política exterior argentina: De Menem a Kirchner, en Relaciones Internacionales, 27, Universidad Nacional de La Plata, Argentina, pp.39-58

BERNAL-MEZA, R. (2013). Modelos o esquemas de integración y cooperación en curso en América Latina (UNASUR, Alianza del Pacífico, ALBA, CELAC): una mirada panorámica, en Ibero-Online.de, Instituto Ibero-Americano, Alemania 
BUSSO, A. (2016). Los ejes de la acción externa de Cristina Fernández: ¿cambios hacia un nuevo horizonte o cambios para consolidar el rumbo?, en Relaciones Internacionales, 50, Facultad de Ciencias Jurídicas y Sociales, Universidad Nacional de La Plata, pp. 143-170

BUSSO, A., ACTIS, E., y NOVELlO, M. R. (2017). La geometría de la Política Exterior Argentina (1989-2015). Fin del diseño triangular e irrupción de un nuevo vértice: las relaciones con Estados Unidos, Brasil y China, en BUSSO, A. (coord.) Modelos de desarrollo e inserción internacional. Tomo 2, UNR, Rosario

CIFRA (2015). La naturaleza política y la trayectoria económica de los gobiernos kirchneristas, Documento de trabajo, 14, Buenos Aires, CTA

CORIGLIANO, F. (2011). La política exterior del gobierno de Cristina Fernández de Kirchner: una típica política peronista del siglo XXI, en Mural Internacional, II (1), junio, Programa de Pós-Graduação em Relações Internacionais da Universidade do Estado do Rio de Janeiro, Brasil, pp.22-27

DE LA BALZE, F. (2010). La política exterior de los gobiernos Kirchner (2003-2009), en Estudios Internacionales, 166, Instituto de Estudios Internacionales, Universidad de Chile, pp.121-140

FUKUYAMA, F. (1989). The end of History?, en The National Interest, 16, Summer, pp. 3-18

GRAMSCI, A. (2003). Notas sobre Maquiavelo, sobre la política y sobre el Estado moderno, Nueva Visión, Buenos Aires

HARVEY, D. (2004). El 'nuevo' imperialismo: acumulación por desposesión, en Socialist register, CLACSO, Buenos Aires

LECHINI, G. y GIACCAGLIA, C. (2016). Introducción, en LECHINI y GIACCAGLIA (eds.) Poderes emergentes y Cooperación Sur-Sur: perspectivas desde el Sur Global, UNR, Rosario

MORAIS, L. e SAAD-FILHO A. (2011). Da economia política à política econômica: o novodesenvolvimentismo e o governo Lula, Revista de Economia Política, 31 (4) (124), pp. 507-527, outubro-dezembro

MORASSO, C. (2016). La orientación autonomista de la política exterior argentina (20032015), en Cuadernos de Política Exterior Argentina (Nueva Época), 123, enero-junio, CERIR, Rosario, pp. 3-22

O'DONNELL, G. (1978). Apuntes para una teoría del Estado, en Revista Mexicana de Sociología, 40 (4), Estado y Clases Sociales en América Latina (2) (Oct. - Dic.), México DF, pp. 1157-1199

POULANTZAS, N. (1981). Las clases sociales en el capitalismo actual, Siglo XXI, México

REGALADO, Roberto. (2014). Guerra de posiciones en América Latina, Ponencia presentada en el XVIII Seminario Internacional Los Partidos y una Nueva Sociedad, México

RUSSEL, R. y TOKATLIAN, J. G. (2002). De la autonomía antagónica a la autonomía relacional: una mirada teórica desde el Cono Sur, en Perfiles Latinoamericanos, 21, Revista de la Facultad Latinoamericana de Ciencias Sociales, México

SCHUTTE, G. R. (2013). O novo desenvolvimentismo e os desafios do pré-sal, Nueva Sociedad especial em português, setembro, pp.76-87

SIMONOFF, A. (2009). Regularidades de la Política Exterior de Néstor Kirchner, en CONfines de Relaciones Internacionales y Ciencia Política, 5 (10), agosto-diciembre, Instituto Tecnológico y de Estudios Superiores de Monterrey México, pp. 71-86

SIMONOFF, A. (2013). Análisis estructural de la estrategia de inserción internacional del primer gobierno de Cristina Fernández (2007-2011), en Relaciones Internacionales, 44, Facultad de Ciencias Jurídicas y Sociales, Universidad Nacional de La Plata, pp. 147-172 
TOKATLIAN, J. G. (2009). Incertidumbres y encrucijadas para la política exterior de Argentina. Notas sobre el escenario internacional y la dinámica regional, Fundación Friedrich Ebert, Argentina

VARESI, G. A. (2011). Argentina 2002-2011: Neo-desarrollismo y radicalización progresista, en Realidad Económica, 264, IADE, Buenos Aires

VARESI, G. A. (2014). La construcción de la hegemonía kirchnerista en Argentina, 2003-2007, en Temas y Debates, 28, Facultad de Ciencia Política y Relaciones Internacionales, Universidad Nacional de Rosario, Argentina

VARESI, G.A. (2018). De la crisis del régimen neo-desarrollista y la hegemonía kirchnerista al triunfo de Cambiemos, en Temas y Debates, 35, Revista universitaria de Ciencias Sociales de la Facultad de Ciencia Política y Relaciones Internacionales de la Universidad Nacional de Rosario, Argentina

\section{Discursos (por orden cronológico)}

Kirchner, N. 25/5/2003. "Discurso de Kirchner ante la Honorable Asamblea Legislativa el día de su asunción presidencial".

Kirchner, N. 18/9/2003. "Cumbre de Jefes de Estado del MERCOSUR, Bolivia y Chile".

Kirchner, N. 25/9/2003. "Discurso de Néstor Kirchner en la ONU, 2003".

Kirchner, N. 13/1/2004. "Desendeudamiento: Néstor Kirchner en la Cumbre Extraordinaria de las Américas en Monterrey".

Kirchner, N. 21/9/2004. "Discurso de Néstor Kirchner en la ONU, 2004".

Kirchner, N. 4/11/2005. "ALCA: Néstor Kirchner en la IV Cumbre de las Américas en Mar del Plata".

Kirchner, .. 15/12/2005. "Discurso de Néstor Kirchner sobre el pago al FMI".

Kirchner, N. 20/9/2006. "Discurso de Néstor Kirchner en la ONU, 2006".

Kirchner, N. 1/3/2007. "Néstor Kirchner en la apertura de sesiones del Congreso, 2007".

Fernández, C. 10/12/2007. "Asunción de Cristina Kirchner, 10 de diciembre de 2007”

Fernández, C. 1/7/2008. "Discurso de la presidenta Cristina Fernández de Kirchner en el acto de apertura de la XXXV Cumbre del Mercosur".

Fernández, C. 23/9/2008. "Discurso de Cristina Kirchner en la ONU, 2008".

Fernández, C. 1/3/2009. "Mensaje de Cristina a la Asamblea Legislativa 2009".

Fernández, C. 3/9/2011. "CFK en la Cumbre del G-20 en Cannes".

Fernández, C. 25/9/2012. "Discurso de la presidenta Cristina Fernández de Kirchner en la $67^{\circ}$ Asamblea General de Naciones Unidas".

Fernández, C. 1/3/2013. "Mensaje de Cristina a la Asamblea Legislativa, 2013".

Fernández, C. 24/9/2014. "Discurso de Cristina Kirchner en la ONU, 2014”. 